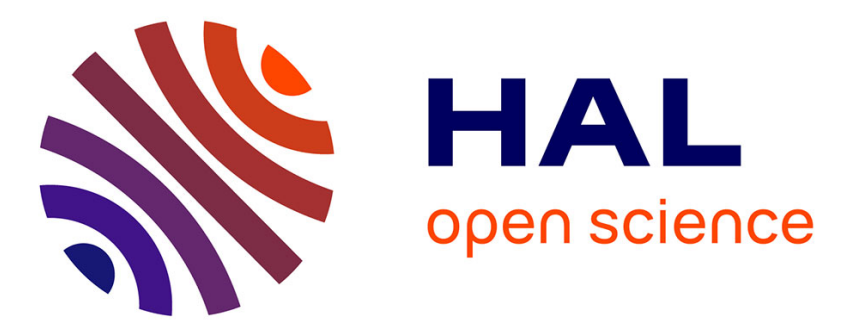

\title{
Mueller micropolarimeter for color imaging of aluminium metasurfaces
}

M. Nicolas, I. Soumahoro, L. Zhang, G. Guida, W. Daney de Marcillac, D. Demaille, C. Schwob, S. Boujday, Bruno Gallas

\section{- To cite this version:}

M. Nicolas, I. Soumahoro, L. Zhang, G. Guida, W. Daney de Marcillac, et al.. Mueller micropolarimeter for color imaging of aluminium metasurfaces. Journal of the Optical Society of America B, 2021, 38 (4), pp.1184. 10.1364/JOSAB.416833 . hal-03178348

\section{HAL Id: hal-03178348 \\ https://hal.science/hal-03178348}

Submitted on 23 Mar 2021

HAL is a multi-disciplinary open access archive for the deposit and dissemination of scientific research documents, whether they are published or not. The documents may come from teaching and research institutions in France or abroad, or from public or private research centers.
L'archive ouverte pluridisciplinaire HAL, est destinée au dépôt et à la diffusion de documents scientifiques de niveau recherche, publiés ou non, émanant des établissements d'enseignement et de recherche français ou étrangers, des laboratoires publics ou privés. 


\title{
Mueller micropolarimeter for color imaging of aluminium metasurfaces
}

\author{
M. Nicolas ${ }^{1,2}$, I. Soumahoro ${ }^{3}$, L. Zhang ${ }^{1,2}$, G. Guida ${ }^{4}$, W. Daney de Marcillac ${ }^{1}$, D. Demaille ${ }^{1}$, C. \\ Schwob $^{1}$, S. Boujday ${ }^{2}$, and B. Gallas ${ }^{1, *}$ \\ ${ }^{1}$ Sorbonne Université, Faculté des Sciences, CNRS, Institut des Nano-Sciences de Paris (INSP), 75005 \\ Paris, France \\ ${ }^{2}$ Sorbonne Université, Faculté des Sciences, CNRS, Laboratoire de Réactivité de Surface (LRS), 75005 \\ Paris, France \\ ${ }^{3}$ Université Nangui Abrogoua, Department of Fundamental and Applied Sciences LPFA, Abidjan, Côte \\ d'lvoire \\ ${ }^{4}$ LEME, EA 4416, Université Paris Nanterre, Nanterre, France \\ ${ }^{*}$ Corresponding author: bruno.gallas@insp.jussieu.fr
}

March 16, 2021

\begin{abstract}
Many anticounterfeiting tags rely on images intricate in different color and/or different polarization states to create optical effects invisible in conventional observation conditions. We build here an imaging micropolarimeter based on the dual rotating compensator system and using the color capabilities of cameras to yield the full polarimetric information for any color coordinates in one acquisition step. After correction for the camera response, the full color image reconstructed from the polarimetric response is validated by the comparison with images obtained in an optical microscope fitted with polarizers.
\end{abstract}

\section{Introduction}

Nanostructures host localized resonances that allow strong interaction with light in a defined wavelength range of the spectrum. This effect has been studied intensively and has been proposed to yield colorful effects with subwavelength resolution that would not fade over time. One can yield these colors with localized surface plasmon resonances [1], holograms [2], diffraction and interferences $[3,4]$, Mie resonances $[5,6,7]$. In addition, the dependence on polarization of anisotropic nanostructures can be added to yield additional effects like encoding images in polarization $[8,9,10,3]$. Different metals exhibit plasmonic resonances but aluminium is particularly interesting thanks to its plasmonic resonance spectrum which covers all the visible range and because it is a low-cost material $[11,12,13,14,15]$. One application of structural and plasmonic colors is in anti-counterfeiting where the ability to build complex structures with unique but easily detectable responses is important. Metasurfaces and nano-resonators have been studied as a way to yield anti-counterfeiting tags $[16,17]$. Finally, some realizations have used polarization effects at one wavelength only [10], however the use of colorful effect allows fancier tags and more importantly different images at different polarization states and colors to be encoded in the same surface. In the design of such tags, having measurement tools combining the full polarimetric properties together with colorimetric coordinates would be particularly interesting.

The most comprehensive polarimetric response of the anticounterfeiting tags is required as it would allow evaluating the intensity observed for any emerging polarization of a surface illuminated with any incident polarization state. In particular, the information about partially polarized light is important since practical observation of many anti-counterfeit tags would use ambient light, which is non-polarized, as a light source. Obviously, anti-counterfeit tags contain an image that can be recognized by the wary observer and would warrant the authenticity of the tag. During the design of such optical effects, it is then indispensable to combine both polarimetric and imaging capabilities to evaluate the Mueller matrix, which contains all the polarimetric information, at different wavelengths of the image under investigation.

Imaging polarimeters have been proposed in particular for biological diagnosis applications using high numerical aperture objectives [18, 19]. High resolution polarimetric imaging of the high harmonic response of a metasurface has also been described [20]. In these cases, the polarization state generator and analyzers are based on non moving elements for stability sake. However, this makes calibration steps and acquisition cumbersome and multi-wavelength imaging difficult. In contrast, devices based on dual-rotating compensators have been the early experimental setups proposed for real time and spectroscopic full Mueller matrix determination in the far field [21, 22, 23]. More recently, imaging using spectrally filtered light has been demonstrated using dual rotating elements [24].

In this work we extend the previous studies made on polarimetric imagers to realize an imaging micro-polarimeter based on a dual rotating compensator configuration and using the color properties of a camera to obtain simultaneously both full polarimetric and color information on the same metasurface in one acquisition run. We illustrate the capabilities of our experimental setup to evaluate the polarization dependence 
of the observed color using a palette containing aluminium nanostructures.

\section{Microscopy polarimetric imaging}

The Mueller-Stokes formalism allows describing the polarimetric response of any surface illuminated by a light source with any polarization state, including unpolarized light. The polarization state of the light is described by the 4-elements Stokes vector $S=\left(I_{t o t}, I_{x}-I_{y}, I_{+45^{\circ}}-I_{-45^{\circ}}, I_{R H P}-I_{L H P}\right)$, where : $I_{\text {tot }}$ is the total intensity; $I_{x}$ and $I_{y}$ are the intensity of the components linearly polarized along directions $x$ and $y ; I_{+45^{\circ}}$ and $I_{-45^{\circ}}$ are the intensity of the components linearly polarized at $+45^{\circ}$ and $-45^{\circ}$ from the $x$ direction; $I_{R H P}$ and $I_{L H P}$ are the intensities of of the components of the right handed and left handed circular polarizations. The $4 \times 4$ Mueller matrix $M$ relates the emerging light described by its Stokes vector $S_{\text {out }}$ to the incident one described by $S_{i n c}$ through: $S_{\text {out }}=M . S_{\text {inc }}$. The determination of the elements of the Mueller Matrix of the samples requires generating different polarization states and analyzing the components of the emerging polarization state. With that aim, we have used a home-built imaging micropolarimeter based on a dual-rotating compensator polarimeter as presented in Figure 1 (a) and inspired from previous works [24]. The Polarization State Generator (PSG) is composed of a linear polarizer and a rotating quarter waveplate yielding different states of polarization of light. The Polarization State Analyser (PSA) is composed of the same elements in the reverse order allowing to analyse any polarization state. With this assembly, the incident polarization is only defined by the linear polarizer of the PSG and, provided that the detector after the PSA is polarization independent, all the needed information about polarization is between the PSG and PSA. The sample to be studied is placed between a condensing lens and a microscope objective in order to image it.
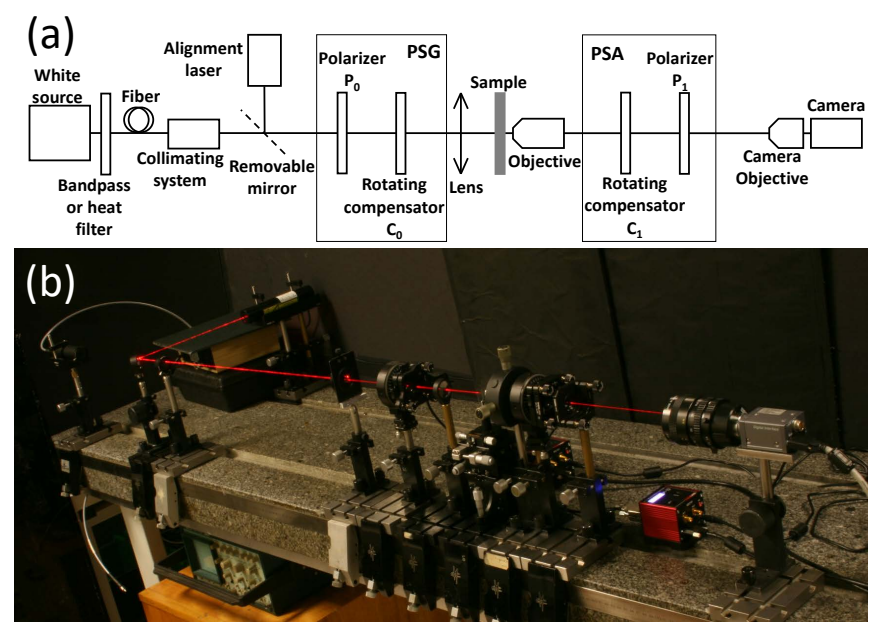

Figure 1: (a) Scheme and (b) photography of the optical setup

\subsection{Method of analysis}

From the time dependant intensity of each pixel of the imaging system, it is possible to extract the full Mueller matrix of each point of the imaged surface. The Stokes vector representing the light seen by the camera $S_{\text {out }}(t)$ is described as a function of the Mueller matrices of the linear polarizers $\left(P_{0}\right.$ and $P_{1}$ oriented at angles $\alpha_{0}$ and $\left.\alpha_{1}\right)$, the two compensators $\left(M_{C_{0}}\right.$ and $\left.M_{C_{1}}\right)$, the objective $M_{o b j}$, the condensing lense $M_{\text {lens }}$, the Mueller Matrix of the sample considered $M_{S}$ (to be determined) and the incoming unpolarized light $S_{i n c}$. The values of $S_{\text {out }}(t)$ vary with time $t$ owing to the rotating compensators, the time clock being given by the framerate of the camera. Considering the order of the different elements (Figure 1 (a)), the intensity measured can be cast in a Stokes vector as follow :

$$
\begin{array}{r}
S_{\text {out }}(t)=P_{1}\left(\alpha_{1}\right) M_{C_{1}}\left(\omega_{1} t+\phi_{1}, \delta_{1}\right) M_{\text {obj }} M_{S} M_{\text {lens }} \\
M_{C_{0}}\left(\omega_{0} t+\phi_{0}, \delta_{0}\right) P_{0}\left(\alpha_{0}\right) S_{\text {inc }}
\end{array}
$$

with $\omega_{0}$ (velocity of $C_{0}$ ), $\omega_{1}$ (velocity of $C_{1}$ ), $\phi_{0}$ (phase at origin of $C_{0}$ ), $\phi_{1}$ (phase at origin of $C_{1}$ ), $\delta_{0}$ (retardance of $\left.C_{0}\right), \delta_{1}$ (retardance of $C_{1}$ ) and time $t$. With unpolarized incident light, $S_{i n c}=(1,0,0,0)$, and without objective or lens, the intensity measured with the camera $I(t)$ is:

$$
I(t)=f\left(\omega_{0}, \omega_{1}, \phi_{0}, \phi_{1}, \delta_{0}, \delta_{1}, \alpha_{0}, \alpha_{1}, t\right) M_{S}
$$

By inverting this formula with a method first used in Ref. [25], we obtain every Mueller matrix element of the sample $M_{S}$.

The angle $\alpha_{0}$ is set to $0^{\circ}$ and all the angles hereafter are determined with respect to $\alpha_{0}$. To determine the other parameters $\left(\alpha_{1}, \phi_{0}, \phi_{1}, \delta_{0}\right.$ and $\left.\delta_{1}\right)$, a calibration procedure is needed. The calibration is made in straight through configuration without sample, lens or objective, so $\left[M_{S}, M_{l e n s}\right.$, $\left.M_{o b j}\right]=\left[I_{4}\right]$ are the identity matrices. It is made by averaging the intensity of all the pixels of the camera, considered as a point detector, and fitting the obtained total intensity to a theoretical curve. In the following, we have assumed that the different optical elements could be described by the Mueller matrices of perfect polarizers and retarders. The time evolution of the total intensity in straight through configuration is then given by :

$$
\begin{array}{r}
I(t)=I_{o}\left(1+C_{2 \alpha_{1}}\left[-\left(C_{\delta_{0}} S_{2 \theta_{0}}^{2}+C_{2 \theta_{0}}^{2}\right)\left(C_{\delta_{1}} S_{2 \theta_{1}}^{2}+C_{2 \theta_{1}}^{2}\right)-\right.\right. \\
\left.\left(1-C_{\delta_{0}}\right)\left(1-C_{\delta_{1}}\right) C_{2 \theta_{0}} S_{2 \theta_{0}} C_{2 \theta_{1}} S_{2 \theta_{1}}+S_{\delta_{0}} S_{\delta_{1}} S_{2 \theta_{0}} S_{2 \theta_{1}}\right]+ \\
S_{2 \alpha_{1}}\left[-\left(1-C_{\delta_{0}}\right) C_{2 \theta_{0}} S_{2 \theta_{0}}\left(S_{2 \theta_{1}}^{2}+C_{\delta_{1}} C_{2 \theta_{1}}^{2}\right)+\right. \\
\left.\left.\left(1-C_{\delta_{1}}\right)\left(-C_{\delta_{0}} S_{2 \theta_{0}}^{2}-C_{2 \theta_{0}}^{2}\right) C_{2 \theta_{1}} S_{2 \theta_{1}}-S_{\delta_{0}} S_{\delta_{1}} S_{2 \theta_{0}} C_{2 \theta_{1}}\right]\right)
\end{array}
$$

where we have used the notations $C_{Y} \equiv \cos (\mathrm{Y}) ; S_{Y} \equiv \sin (\mathrm{Y})$; $\theta_{0}=\omega_{0} \mathrm{t}+\phi_{0}$ and $\theta_{1}=\omega_{1} \mathrm{t}+\phi_{1}$. The prefactor $I_{o}$ is the total intensity reaching the detector and is used to normalize $\mathrm{I}(\mathrm{t})$ in the determination of the Mueller matrices.

It can be noted that with $\alpha_{1}=90^{\circ}$ we obtain the relation given in Ref [24]. Once the calibration of the angles and retardance of the waveplates done, a correction thanks to residual retardation introduced by the imaging system (lens + objective) has to be performed for every pixel. Using equation 2 with $M_{S}=\mathrm{I}_{4}$ (so without sample), we determine $M_{o b j} M_{l e n s}$. We can not separate experimentally $M_{o b j}$ and $M_{\text {lens }}$ although the sample is placed between the two. To apply the correction, we consider $M_{o b j} M_{\text {lens }}$ as a retarder with a small retardance (which is confirmed experimentally in Figure 2 (a) 
and (b)). So, $M_{o b j} M_{l e n s}$ can be approximated to a diagonal matrix. We then assumed that $M_{S}$ and $M_{\text {lens }}$ commute with $M_{S}$. Finally, we can consider a corrected matrix for the sample which is defined by:

$$
M_{S}^{\text {corrected }}=\left(M_{o b j} M_{l e n s}\right)^{-1} M_{S}^{\text {measured }}
$$

\subsection{Characteristics of the setup}

A photograph of our experimental setup illuminated with the alignment laser is presented in Figure 1 (b). The white source was a Stabilized Broadband Light Source (Thorlabs - SLS201/M) fitted with a fiber coupler. The beam was filtered through a bandpass or heat filter. The heat filter (Heat Abs Glass KG-5, Edmunds Optics) was used for measurements with white illumination in order to only consider the visible part of the light spectrum. The monochromatic measurements were performed with Corion bandpass filters with a bandwidth of $70 \mathrm{~nm}$. In the following we will only mention the center wavelength of the filters. The light was condensed at the output of the fiber to yield a collimated parallel beam. The Polarization State Generator (PSG) and the Polarization State Analyser (PSA) were composed of fixed linear polarizers (PGT-S1V Glan-Taylor polarizer and WP25M-UB Wire grid polarizer Thorlabs, respectively) and super achromatic quarter wave plates as compensators (SAQWP05M-700 superachromatic quarter waveplates Thorlabs). A plan-convex converging lens $(\mathrm{f}=75 \mathrm{~mm})$ was used to condense the incident beam and increase the illuminance on the surface of the sample. It was placed after the PSG so that the incident beam on the PSG is collimated. An infinite conjugated objective (Olympus, UMPIanFI $20 \times / \mathrm{NA}=0.46$ ) was used to image the surface. The camera was a Sony XCD-SX90CR allowing an acquisition of 30 frames per second fitted with an objective with $69 \mathrm{~mm}$ of focal length.

We crossed the polarizer and the analyser at $\alpha_{0}=0^{\circ}$ and $\alpha_{1}=90^{\circ}$ since the relative angles are easy to set thanks to Malus' law.

The compensators were integrated into motorized rotation stages (Thorlabs PRM1Z8 and Thorlabs KDC101 K-Cube Brushed DC Servo Motor Controller). These rotation stages permit to control the position and rotation speed of the compensators at every time up to $25^{\circ} . \mathrm{s}^{-1}$ with an accuracy of $0.1 \%$. The compensators were rotating at $\omega_{0}=4^{\circ} . \mathrm{s}^{-1}$ and $\omega_{1}=20^{\circ} . \mathrm{s}^{-1}$, respectively, with fixed phases at origin $\phi_{0}$ and $\phi_{1}$ corresponding to the mechanical misalignment of the fast axis of the waveplates with the reference of the motor angle with respect to $\alpha_{0}$.

The velocity ratio of the compensators is of crucial importance in order to allow the extraction of the Mueller matrix of the sample from the measured intensities. Following previous studies, we chose a ratio of $\frac{\omega_{0}}{\omega_{1}}=5[24,22,23,26]$. To achieve a measurement, a single turn of the slowest compensator is needed, i.e. 90 seconds. To ensure that the values of $\phi_{0}$ and $\phi_{1}$ were always the same after calibration, the motors were synchronized with the camera so that the image acquisition and angle readings referred to the same clock. After the calibration procedure described previously, we obtained the values of the five angles : $\alpha_{1}, \phi_{0}, \phi_{1}, \delta_{0}$ and $\delta_{1}$

We have to consider the systematic errors that arise from the non-ideality of the different optical elements, uncertainties about the calibration results and uncertainties about the alignment procedure. The position of the analyser has been determined thanks to Malus' law, we find, with the calibration fit, $\alpha_{1}=90 \pm 10^{-2}$. Since the compensators are not perfectly achromatic, the values for the retardances were determined for each wavelength used during the experiments. We have used a conventional ellipsometer to cross-check the values found with our calibration procedure. For instance, the value of the retardance of the compensator $C_{1}$ varied around $94^{\circ}+/-2^{\circ}$ in the $400 \mathrm{~nm}-800 \mathrm{~nm}$ spectral range. With a filter centered at $620 \mathrm{~nm}$, we found with our calibration $\delta_{1}=92.97^{\circ}$ while with ellipsometric measurement $\delta_{1}=92.70^{\circ}$ at $620 \mathrm{~nm}$. This shows that the calibration procedure yields accurate values of the retardance for our compensators. There is also a systematic uncertainty about the position of the compensators. Indeed, the fastest component is rotating at $20^{\circ} . \mathrm{s}^{-1}$ whereas the framerate of the camera is $30 \mathrm{~s}^{-1}$. This induces a $0.67^{\circ}$ systematic uncertainty in the position of this rotating element. The propagation of errors has been studied and can be found in Refs.[24, 27, 28]. With a first order approximation, we estimate that the systematic errors of our setup introduces an uncertainty for the Mueller matrix coefficients to be generally below \pm 0.02 except for the elements $\mathrm{M}_{23}$ and $\mathrm{M}_{32}( \pm 0.05)$ which are more sensitive to errors [24].

Random noise affects also the values of the Mueller matrix elements. The dispersion of the data in each pixel of the camera can be automatically reduced thanks to an averaging of several consecutive measurements. In order to analyze this influence, a measurement has been performed with a filter centered in $650 \mathrm{~nm}$. The dispersion of the matrix coefficients of the imaging system $M_{o b j} M_{l e n s}$ in air averaged over the whole imaged field are shown in Figure 2(a).

The standard deviations were of $5.10^{-3}$ after averaging five consecutive measurements. To extract the polarization properties of the imaging system we have used the logarithmic decomposition of the Mueller matrix $M_{o b j} M_{l e n s}[29,30]$. The imaging field was $150 \times 150$ pixels $^{2}$. The average depolarization extracted over all the pixels was of 0.047 on the average and the polarizance $\bar{P}$ was found nearly equal to zero with an average value of 0.015 (inset of Figure 2(b)). These values hint towards the imaging system behaving as a pure retarder. Figure 2(b) presents the retardance map obtained with the logarithmic decomposition. It was homogeneous across the camera detector and had an average value of $-1.03^{\circ}$. This justifies a posteriori the approximations made in our correction model (Eq. 4) considering that the imaging system could be seen as a linear retarder with a small retardance.

\subsection{Precession of the rotating compensators}

When imaging microscopic objects, one source of error induced by this system is the effect of the rotating element on the position of the beam emerging from the PSA. An error which can not be reduced originates in the faces of the output compensators not being perfectly parallel resulting in a 
(a)
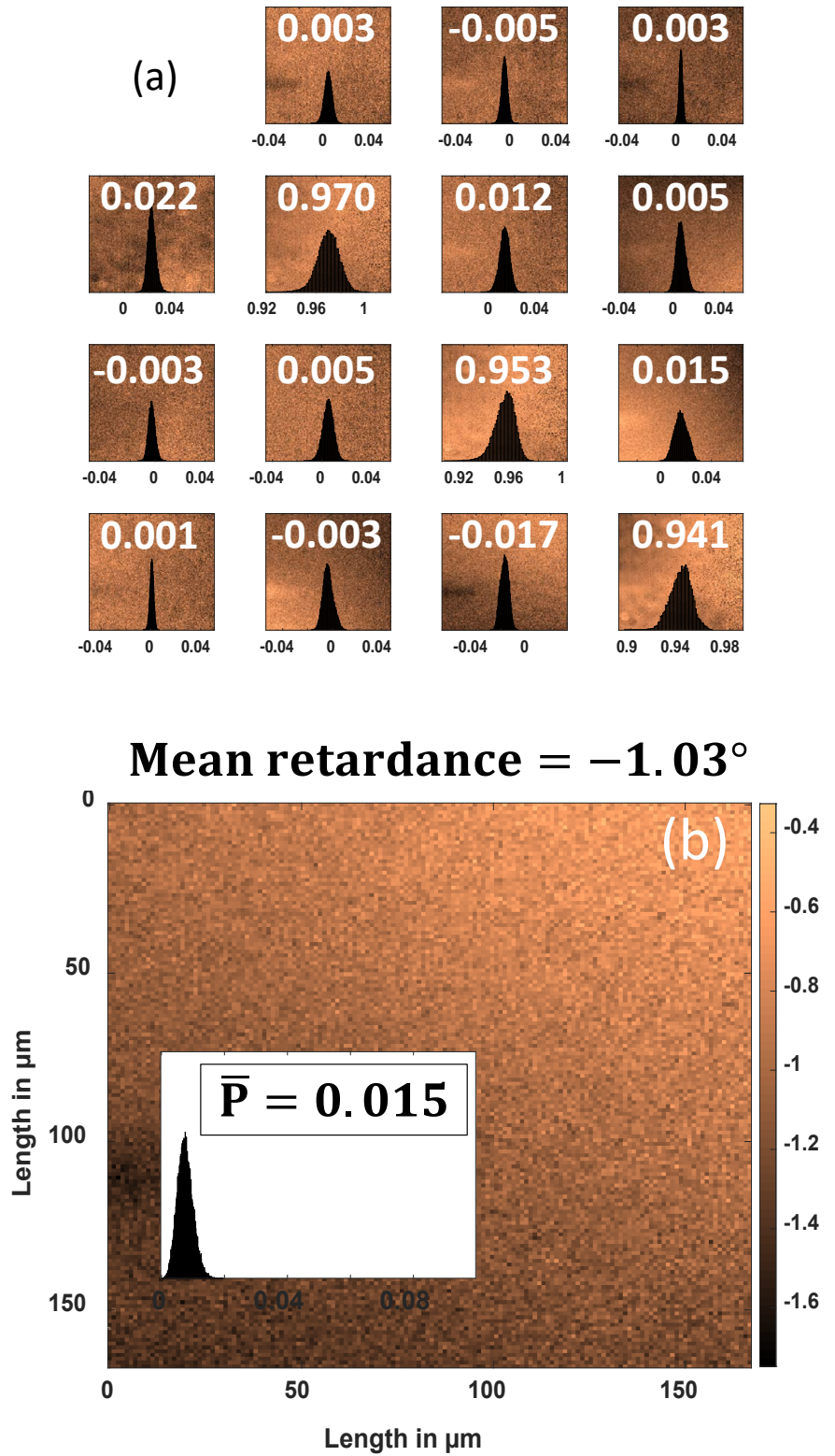

Figure 2: (a) Mueller matrix $M_{o b j} M_{\text {lens }}$ of the imaging system before correction after averaging five consecutive measurements at $650 \mathrm{~nm}$. The insets present the histograms of the values distribution over all pixels in each element with the mean values. (b) Image of the spatial variation of the retardance of imaging system modeled as a pure retarder. The inset presents the histogram of the polarizance values over all the pixels of the camera of the imaging system.

deviation of the beam. A point of the sample will no longer be imaged on the same pixel of the camera yielding a blurred image. The waveplates used here are given by the constructor with a beam deviation below 3 arcmin (around $5.10^{-2} \circ$ ). This deviation would be well below the acceptance angle of the wire grid polarizer $\left(20^{\circ}\right)$ which ensures that the intensity detected on the detector would allow extracting the Mueller matrix elements. Our PSA is located at approximately 30 $\mathrm{cm}$ from the camera detector but the focal length of the camera objective is $69 \mathrm{~mm}$ which is the actual length to be taken into account. This focal length would result in a displacement of the image of approximately $60 \mu \mathrm{m}$ on the CCD detector of the camera, across a full rotation of the compensator of the PSA. This effect would strongly hamper the resolution of the imaging system. Before placing the waveplate in our micro-polarimeter, we have determined, experimentally, the beam deviation induced by the non-parallel faces of the compensator and found it to be below approximately $5 \cdot 10^{-3}{ }^{o}$. This results in a displacement of the image on the detector plane below $6 \mu \mathrm{m}$. This value must be compared to the size of the sub-pixels of our camera detector which are $3.75 \times 3.75$ $\mu \mathrm{m}^{2}$ organized in the $2 \times 2$ Bayer configuration. In the color imaging configuration, we will consider only 1 sub-pixel out of four for each color. So, two sub-pixels of the same color are actually separated by to $7.5 \mu \mathrm{m}$. The expected displacement of the image should be of approximately 1 pixel for our resolution. This influence is shown in Figure 3 where the Mueller Matrix element $M_{43}$ determined at $650 \mathrm{~nm}$ of a metasurface representing a Mona Lisa made of gold U-shaped resonators [10] is compared to the intensity image measured on the same surface with the compensators not rotating (Figure 3 (a)). The image is $100 \times 100 \mu \mathrm{m}^{2}$ with $90 \times 90$ points : we can see that fine details can still be resolved despite the possible displacement of the beam induced by the rotating compensators.
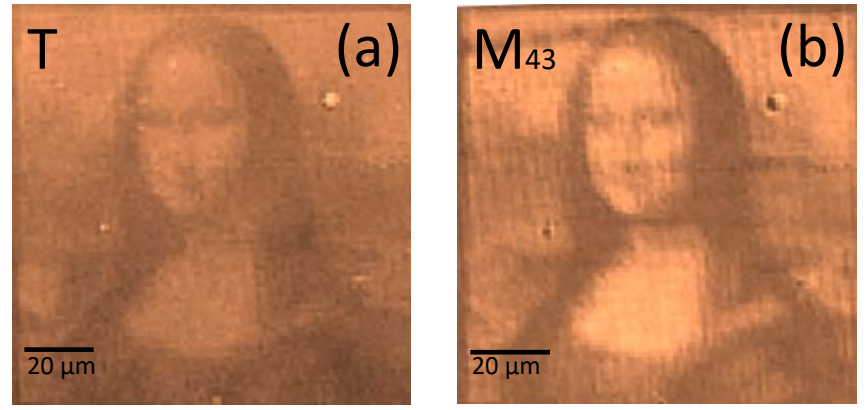

Figure 3: (a) Image of a metasurface containing a reproduction of Mona Lisa in direct imaging configuration of the micro-polarimeter (transmittance T) and (b) $M_{43}$ coefficient of the Mueller matrix determined after measurement at 650 $\mathrm{nm}$. The details of the image remain clearly visible despite the possible displacement of the beam induced by the rotating elements in the micro-polarimeter.

The beam deviation error may be reduced by applying a position correction of the image by tracking computationally the position of the image as a function of the waveplate angle. However, with the magnification used here, the deviation had more or less the same magnitude as a pixel resolution and was not necessary.

\subsection{Use of Mueller matrix to obtain the col- orimetric coordinates of a sample}

To achieve a polarimetric measurement with color information, the signal obtained at three different wavelengths corresponding to the red $(\mathrm{R})$, green $(\mathrm{G})$ and blue $(\mathrm{B})$ colorimetric coordinates could be enough thanks to the possibility to make RGB reconstructions. With our system, a feasible solution would be to measure our sample with three different band-pass filters placed in front of the broadband light 
source. However, it is possible to use the properties of the detector of our color camera for which each pixel contains 4 sub-pixel arranged in a Bayer configuration [31]. The Bayer configuration within each pixel is presented in Figure 4(a) together with the spectral response of the color filters placed in front of each sub-pixel (Figure 4(b) is a graph taken from the constructor notice). These filters effectively achieve the spectral filtering of the broadband source and the polarimetric response for each color can be acquired in only one acquisition, provided that each sub-pixel is treated separately. A single white illumination allows 3 measurements at three different wavelengths.

However, it can be seen that the filters have a rather large bandwidth and that the spectral signal overlap. In addition it is known that the blue filter can show some efficiency again in the near infrared, requiring the use of a heat filter. (a)

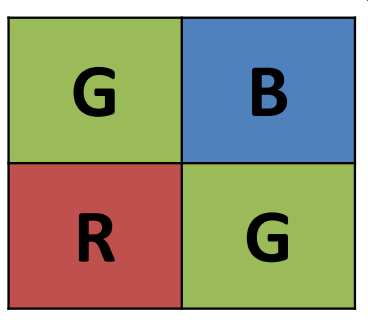

Relative sensitivity
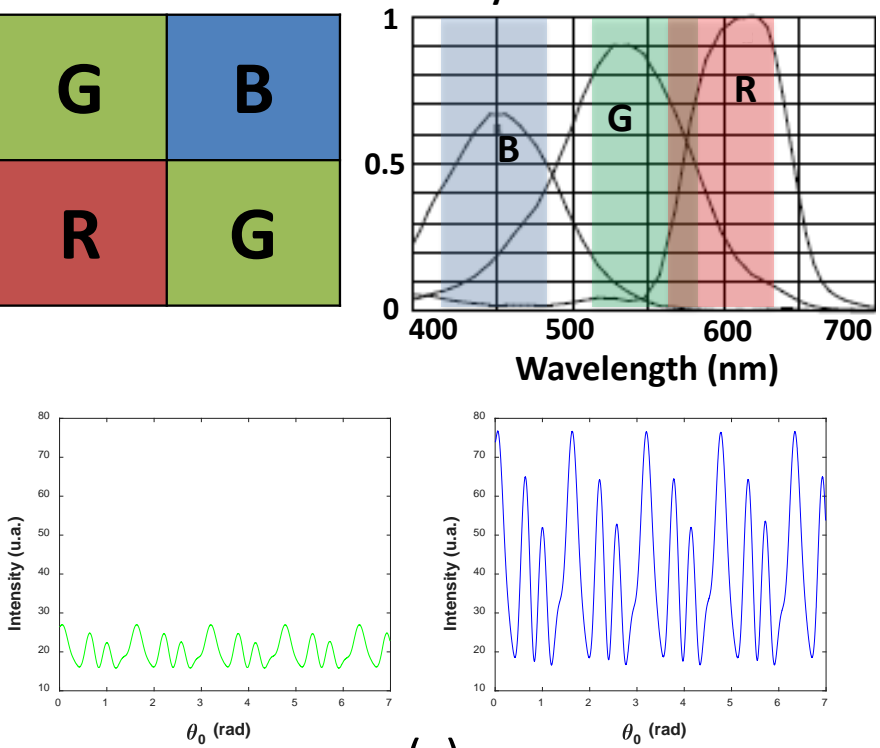

(c)
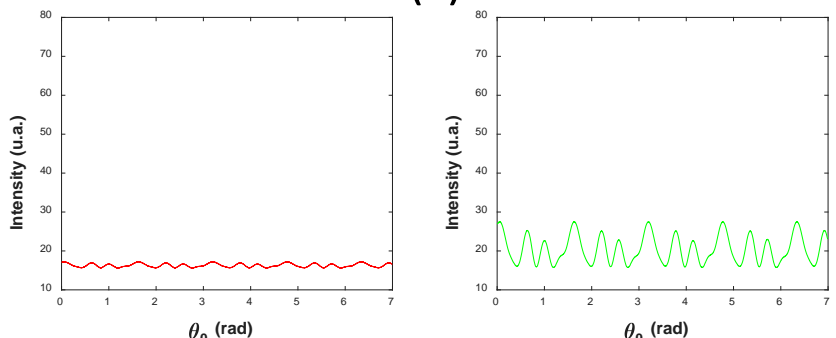

Figure 4: (a) Bayer RGB configuration of the camera. (b) Manufacturer characteristics for the sensitivity of the different RGB pixels (full lines). The positions and bandwidths of the Corion filters are also indicated as colored rectangles. (c) Mean intensity in each sub-pixels of the Bayer configuration with an incoming blue light centered in 450nm.

As a consequence of the overlap of the spectral response of the R, G and B filters of the camera, cross-talk can be expected between the sub-pixels. Figure 4 (c) presents the time dependence of the intensities recorded in each sub-pixel in straight through configuration for an incoming blue light (filtered at the source at $450 \mathrm{~nm}$ ). It is clearly observed that the amplitude of the intensity variations in each sub-pixel of the camera depends on the incoming wavelength. In Figure 4 (c), there was some cross-talk among the pixels, particularly between the $\mathrm{G}$ and $\mathrm{B}$ ones since the intensity in the $\mathrm{G}$ pixels is not negligible. Consequently, three measurements have been made at $\lambda_{B}=450 \mathrm{~nm}, \lambda_{G}=550 \mathrm{~nm}$ and $\lambda_{R}=600 \mathrm{~nm}$, as close as possible to the maximum of the camera filters, in order to obtain all the ratio of the amplitudes characterizing the cross-talk. An $\mathrm{X}$ matrix with $3 \times 3$ dimension containing the cross-talk parameters is presented below with the experimental values (in the equations, the intensities are represented with this formalism : $\left.I_{\text {subpixel }}^{\text {source }}\right)$ :

$$
X=\left[\begin{array}{ccc}
1 & \frac{I_{R}^{\lambda_{G}}}{I_{G}^{\lambda_{G}}} & \frac{I_{R}^{\lambda_{B}}}{I_{B}^{\lambda_{B}}} \\
\frac{I_{G}^{\lambda_{R}}}{I_{R}^{\lambda_{R}}} & 1 & \frac{I_{G}^{\lambda_{B}}}{I_{B}^{\lambda_{B}}} \\
\frac{I_{B}^{\lambda_{R}}}{I_{R}^{\lambda_{R}}} & \frac{I_{B}^{\lambda_{G}}}{I_{G}^{\lambda_{G}}} & 1
\end{array}\right]=\left[\begin{array}{ccc}
1 & 0.24 & 0.03 \\
0.09 & 1 & 0.19 \\
0.01 & 0.07 & 1
\end{array}\right]
$$

An ideal intensity $I_{\text {ideal }}$ is calculated. It contains the three intensities needed to determine the Mueller matrices at the three corresponding wavelengths with the method presented previously ( $\Sigma$ stands for white illumination) :

$$
\left[\begin{array}{c}
I_{R}^{\Sigma} \\
I_{G}^{\Sigma} \\
I_{B}^{\Sigma}
\end{array}\right]=X\left[\begin{array}{c}
I_{R}^{\lambda_{R}} \\
I_{G}^{\lambda_{G}} \\
I_{B}^{\lambda_{B}}
\end{array}\right] \quad \text { and } \quad I_{\text {ideal }}=\left[\begin{array}{c}
I_{R}^{\lambda_{R}} \\
I_{G}^{\lambda_{G}} \\
I_{B}^{\lambda_{B}}
\end{array}\right]=X^{-1}\left[\begin{array}{c}
I_{R}^{\Sigma} \\
I_{G}^{\Sigma} \\
I_{B}^{\Sigma}
\end{array}\right]
$$

Three Mueller matrices normalized to $M_{11}$ are determined : $M_{R}, M_{G}$ and $M_{B}$. There is one matrix for each pixel of the camera. One can uses these matrices by multiplying with an input Stokes Vector at each pixel to simulate the effect of any incoming polarization on a sample. For each pixel of the camera, we obtain an output Stokes vector:

$$
S_{\text {input }}=\left[\begin{array}{c}
S_{1} \\
S_{2} \\
S_{3} \\
S_{4}
\end{array}\right] \quad \text { with }\left\{\begin{array}{l}
S_{\text {output }}^{\lambda_{R}}=M_{R} S_{\text {input }} \\
S_{\text {output }}^{\lambda_{G}}=M_{G} S_{\text {input }} \\
S_{\text {output }}^{\lambda_{B}}=M_{B} S_{\text {input }}
\end{array}\right\}
$$

which describes the polarization state of the output light. Thanks to the Stokes formalism, it is possible to choose to display whether the total intensity, i.e. the first element of $S_{\text {output }}$, or different characteristics of the polarization of the light by using the different components of the Stokes vector. By inserting the Mueller matrices of polarizers in the calculation, it is also possible to filter the response for any polarization of observation. In the following we will focus on using the wavelength dependence information to make a polarized RGB reconstruction of an image.

\section{Application to a metasurface made of nano- $U$ aluminium struc- tures}

A palette containing aluminium nanostructures has been imagined in order to evaluate the polarization dependence 
of the observed colors for these structures. Different sizes of U-shaped resonators placed on a square lattice with different lattice constants were tested. Indeed, it is known that both size and distance between resonators can affect the color observed [15]. With the aim of obtaining red, blue and green colors in transmission, the size of the resonators and the lattice constant were designed using calculations based on Finite Element Methods (HFSS by ANSYS). We used aluminium as material to allow for plasmonic resonances in the blue part of the spectrum to be excited. We obtained that resonators with total footprints of $320 \times 320 \mathrm{~nm}^{2}$ (Figure $5(\mathrm{a})$ ), $270 \times 270$ $\mathrm{nm}^{2}$ (Figure $5(\mathrm{~b})$ ) and $170 \times 170 \mathrm{~nm}^{2}$ (Figure 5(c)) could yield red, green and blue colors respectively. We also investigated four lattice constants of $350 \mathrm{~nm}, 400 \mathrm{~nm}$ (Figure 5), $450 \mathrm{~nm}$ and $500 \mathrm{~nm}$. The samples were made with a classical e-beam lithography (EBL) method. Figure 5 presents a sketch of the different U-shaped resonators investigated together with the Scanning Electron Microscopy (SEM) images of the obtained square lattices of resonators made of aluminium. To obtain these resonators, a $150 \mathrm{~nm}$ thick PMMA layer was spin-coated on a glass substrate followed by a $20 \mathrm{~nm}$ thick coating Electra92 to avoid charging effects during the electron beam exposure. After e-Beam inscription, the Electra resist was removed in deionised water then a MIBK-IPA (mixture of méthylisobutylcétone and isopropanol) development step was made to reveal the exposed areas. A $40 \mathrm{~nm}$ thick aluminium layer was deposited and finally the excess resist was lift-off to yield arrays of U-shaped resonators on the glass substrate. For each combination of resonator size and lattice constant, an area of $20 \times 20 \mu \mathrm{m}^{2}$ was made resulting in 12 areas.

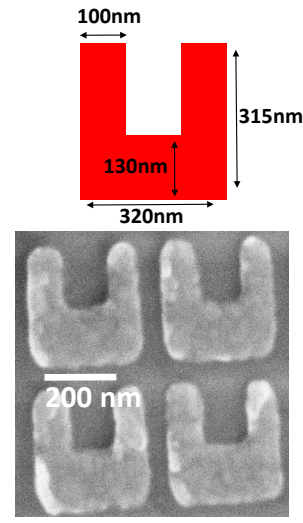

(a)

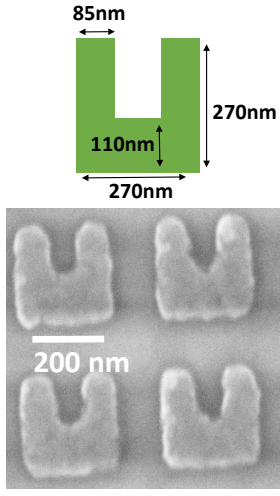

(b)
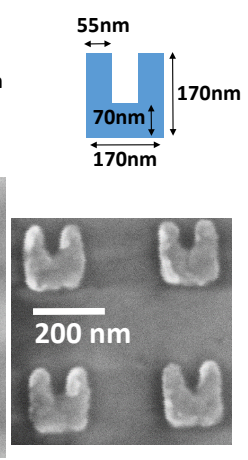

(c)

Figure 5: Scanning Electron Microscopy (SEM) images of Ushaped nano structures of different size scaling on a lattice of $400 \mathrm{~nm}$ : resonators of (a) $320 \mathrm{~nm}$ (b) $270 \mathrm{~nm}$ and (c) $170 \mathrm{~nm}$. The schematics above the images give the actual dimensions.

The different plasmonic resonant modes excited in Ushaped resonators have already been studied extensively $[32,33,34]$. The different modes can be excited selectively with light linearly polarized either along the lateral arms or along the bottom arm of the U-shaped resonators hence yielding different colors. These metasurfaces were observed using a conventional optical microscope (Olympus BX61) fitted with a heat filter and linear polarizers. The observations were made in transmission bright field mode and the

observed images are presented in Figure 6 (a) and (b). The white balance was performed on the light transmitted in the glass next to the areas containing the resonators and set to $(\mathrm{R}, \mathrm{G}, \mathrm{B})=(255,255,255)$. One can observe that each patch had a different color and that the color in each patch changed depending on the state of polarization for the incoming light. Since the Numerical Aperture of the microscope objective may affect the observed colors, the measurements were made with the same $\times 20$ objective as the one used in the micropolarimeter. With this objective, a single measurement is enough to observe the twelve patches. (a)

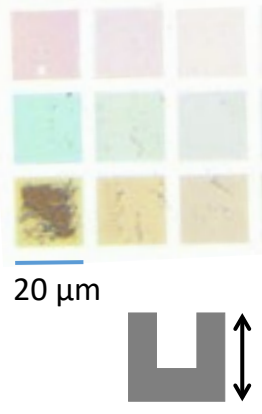

(c)

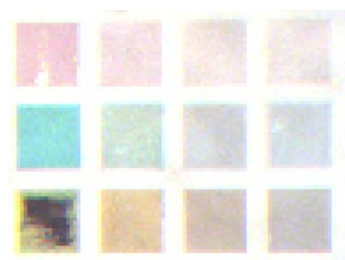

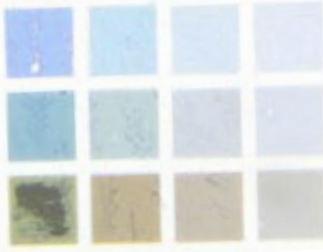

(b)
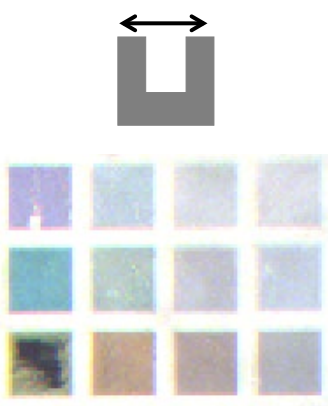

Figure 6: Optical Microscope observations of the samples of Aluminium Nanostructures with two differents states of incoming linearly polarized light respectively (a) vertically and (b) horizontally with corrected backgrounds. RGB recombination of an output Stokes parameter after a single Mueller Matrix measurement for incoming linearly polarized light respectively (c) vertically and (d) horizontally.

The metasurface has been measured also with the polarimetric setup. The Mueller matrices of the sample have been recorded with white heat-filtered illumination at normal incidence. After obtaining these Mueller matrices, the method presented previously to recover the colorimetric coordinates of a sample with specific states of incoming or output polarization has been used. After a cross-talk correction, we chose to use two input Stokes vectors to observe the effect of an incoming vertically and horizontally polarized light, $S_{i n}=(1,1,0,0)$ and $S_{i n}=(1,-1,0,0)$ respectively. The angles of the linear polarizations have been optimized by adjusting the Stokes vector because we can not apply a light perfectly polarized parallely or perpendicularly to the arms of the $U$ resonators. We considered the first component of the output Stokes vector and after an RGB recombination, a color is obtained for each pixel of the image. A white balance was performed by applying for each color a correction calculated by: (i) determining the average value of the intensity $I_{o}$ (Equation 3) over a small area of the glass substrate, $<I_{o, \text { glass }}^{i}>$ with $\mathrm{i}=\left(\lambda_{R}, \lambda_{G}, \lambda_{B}\right)$, and (ii) applying to each pixel and each color channel the multiplicative factor 255.I $I_{o, \text { pixel }}^{i} /<I_{o, \text { glass }}^{i}>$. The reconstructed color images obtained for each incoming polarization state are shown in Figure 6 (c) and (d). We observe a qualitative very 
good agreement between the colors observed in the optical microscope (Figure $6(\mathrm{a})$ and (b)) and the reconstructed ones (Figure 6 (c) and (d)) which supports our methodology for extracting the polarized-resolved color information of metasurfaces at the micrometer scale.

Some improvement may be brought to allow for a comparison using real colorimetric coordinates. The achromaticity of the optical components can not be improved much, in particular for the quarterwave plates. A possibility could be to use a Fresnel Rhomb retarder instead of an achromatic wave plate. Fresnel Rhomb retarder bring improvement in the fast angle orientation compared to the achromatic quarterwave plates for which the fast axis varies between $-1.59^{\circ}$ and $+0.78^{\circ}$ in the visible part of the spectrum. The retardance deviates from a quarterwave by 0.004 wave for a Fresnel Rhomb and by 0.008 wave for the achromatic wave plates the the visible range. However, because the retarders are rotating continuously, the beam deviation is a lot more easier to minimize with in imaging conditions with quarterwave plates. The variation with wavelength of the fast axis and retardance of the quarterwave plates, together with the broad wavelength analyzed in each pixel of the Bayer configuration, should induce depolarization in the measurements. The depolarisation measured remained low. Indeed, using a previous study [35], we have calculated the depolarization induced by the two quarterwave plates in straight through configuration with : depolarization $=1-\sqrt{\frac{\operatorname{tr}\left(M^{t} M\right)-M_{11}^{2}}{3 * M_{11}^{2}}}$ where $M=M_{C_{1}}$ or $M=M_{C_{0}}$ [36]. Considering the spectral dependence of the retardance and fast axis orientation of the achromatic quarterwave plates provided by the manufacturer and with a bandwith of $70 \mathrm{~nm}$, we found a depolarization of $1.5^{*} 10^{-3}, 8.6^{*} 10^{-4}$ and $1.8^{*} 10^{-4}$ at $450 \mathrm{~nm}$, $550 \mathrm{~nm}$ and $600 \mathrm{~nm}$, respectively. These values are well below the value extracted for $M_{o b j} M_{\text {lens }}$ presented above. This shows that the non-ideal retardance of the quarterwave plates was not a limiting factor in our setup. This is in line with the propagation in the Mueller matrices of errors made on the actual value of the retardance [24]. The overlap of the color filters of the CCD detector of our camera induces probably cross-talk corrections slightly different from the ones used here and the values may be retrieved using color standards : this was beyond the scope of this work and the determination of the cross-talk coefficients using filters allowed us to clearly show how it affected the measurements. The interest of the procedure described here is multiple. Many anti counterfeiting tags proposed are using images interleaved in orthogonal polarization states $[3,8,9]$ or hidden in some polarization state [10]. The good contrast between the different images relies on the perfect alignment of the polarization axes used for imaging with the axes of the images. The full polarimetric measurement of the sample and fast post-processing of the data would allow finding directly the optimum polarization axes to have the better contrast or reveal the image without relying on tedious alignment for the end user (be it scientist or customer). For commercial applications, there will be need of polarimetric measurements. There are already Stokes came ras available, generally working at one wavelength. We show here that imaging at multiple wavelengths to render color polarized images would be feasible. There are more and more proposals of using the camera of smartphones for anti counterfeiting applications. Recent publications on metasurfaces allow to envision external adaptors that could be fitted to the camera of smartphones to allow for Stokes imaging [37]. The full color measured Mueller matrix of the tag could be recorded in a secured database and used to validate the measured Stokes vector.

\section{Conclusion}

In this work we have built a micropolarimeter combining imaging and color rendering capabilities. The micropolarimeter was based on a dual rotating compensator system allowing to determine all 16 elements of the Mueller matrix. Thanks to the use of short focal length camera, the error on the beam position introduced by the rotating elements could be maintained at acceptable levels. The color capability was obtained through the use of the Bayer configuration of the detector of the camera to filter the three R, G and B color coordinates. Three Mueller matrices were determined simultaneously after cross-talk correction and allowed reconstructing a color image observed for two orthogonal linear polarizations. Obviously, the mathematical reconstruction could be used for any combination of incident to emerging polarization. Such a polarimeter and the information derived from its measurements could be used to investigate systematically and conveniently the color response for any polarization conditions of anti-counterfeiting tags where images would be intricated in color and polarization.

\section{Funding}

This work has been carried out thanks to the support of the ANR Chiroptmol (n ANR-18-CE09-0010-01). Three authors (L.Z., S.B. and B.G.) acknowledge support by the Cluster of Excellence MATISSE funded by the Investissements d'Avenir French Government program. Part of this work was carried out within a bipartite agreement between Sorbonne University and University Nangui Abrogoua.

\section{Acknowledgments}

The authors acknowledge the helps of F. Breton at the INSP for the interfacing of the micropolarimeter.

\section{Disclosures}

The authors declare no conflicts of interest related to this paper.

\section{References}

[1] Anders Kristensen, Joel K. W. Yang, Sergey I. Bozhevolnyi, Stephan Link, Peter Nordlander, Naomi J. Halas, and N. Asger Mortensen. Plasmonic colour generation. Nature Reviews Materials, 2(1):16088, January 2017.

[2] Yao-Wei Huang, Wei Ting Chen, Wei-Yi Tsai, Pin Chieh Wu, Chih-Ming Wang, Greg Sun, and Din Ping Tsai. 
Aluminum Plasmonic Multicolor Meta-Hologram. Nano Letters, 15(5):3122-3127, May 2015.

[3] Luc Duempelmann, Angélique Luu-Dinh, Benjamin Gallinet, and Lukas Novotny. Four-Fold Color Filter Based on Plasmonic Phase Retarder. ACS Photonics, 3(2):190-196, February 2016.

[4] Ewan D. Finlayson, Ian R. Hooper, Christopher R. Lawrence, Mark Heath, David Anderson, J. Roy Sambles, and Peter Vukusic. Covert Images Using Surface Plasmon-Mediated Optical Polarization Conversion. Advanced Optical Materials, 6(5):1700843, March 2018.

[5] Julien Proust, Frédéric Bedu, Bruno Gallas, Igor Ozerov, and Nicolas Bonod. All-dielectric colored metasurfaces with silicon mie resonators. ACS Nano, 10(8):7761-7767, 2016.

[6] Wenhong Yang, Shumin Xiao, Qinghai Song, Yilin Liu, Yunkai Wu, Shuai Wang, Jie Yu, Jiecai Han, and Din-Ping Tsai. All-dielectric metasurface for highperformance structural color. Nature Communications, 11(1):1864, 2020.

[7] Pengcheng Huo, Maowen Song, Wenqi Zhu, Cheng Zhang, Lu Chen, Henri J. Lezec, Yanqing Lu, Amit Agrawal, and Ting $\mathrm{Xu}$. Photorealistic full-color nanopainting enabled by a low-loss metasurface. Optica, 7(9):1171-1172, Sep 2020.

[8] Mathilde Makhsiyan, Patrick Bouchon, Julien Jaeck, Jean-Luc Pelouard, and Riad Haïdar. Shaping the spatial and spectral emissivity at the diffraction limit. Applied Physics Letters, 107(25):251103, December 2015.

[9] Peter Zijlstra, James W. M. Chon, and Min Gu. Fivedimensional optical recording mediated by surface plasmons in gold nanorods. Nature, 459(7245):410-413, May 2009.

[10] Thomas Sang Hyuk Yoo, Johann Berthelot, Géraldine Guida, Dominique Demaille, Enric Garcia-Caurel, Nicolas Bonod, and Bruno Gallas. Circularly Polarized Images with Contrast Reversal Using Pseudochiral Metasurfaces. ACS Photonics, 5(10):4068-4073, October 2018.

[11] Jeppe S. Clausen, Emil Højlund-Nielsen, Alexander B. Christiansen, Sadegh Yazdi, Meir Grajower, Hesham Taha, Uriel Levy, Anders Kristensen, and N. Asger Mortensen. Plasmonic Metasurfaces for Coloration of Plastic Consumer Products. Nano Lett., 14(8):44994504, August 2014.

[12] Mark W. Knight, Nicholas S. King, Lifei Liu, Henry O. Everitt, Peter Nordlander, and Naomi J. Halas. Aluminum for Plasmonics. ACS Nano, 8(1):834-840, January 2014.

[13] Renilkumar Mudachathi and Takuo Tanaka. Up Scalable Full Colour Plasmonic Pixels with Controllable Hue, Brightness and Saturation. Sci Rep, 7(1):1199, December 2017.
[14] Jana Olson, Alejandro Manjavacas, Lifei Liu, Wei-Shun Chang, Benjamin Foerster, Nicholas S. King, Mark W. Knight, Peter Nordlander, Naomi J. Halas, and Stephan Link. Vivid, full-color aluminum plasmonic pixels. Proc Natl Acad Sci USA, 111(40):14348-14353, October 2014.

[15] Shawn J. Tan, Lei Zhang, Di Zhu, Xiao Ming Goh, Ying Min Wang, Karthik Kumar, Cheng-Wei Qiu, and Joel K. W. Yang. Plasmonic Color Palettes for Photorealistic Printing with Aluminum Nanostructures. Nano Lett., 14(7):4023-4029, July 2014.

[16] Ling Li. Technology designed to combat fakes in the global supply chain. Business Horizons, 56(2):167-177, March 2013.

[17] Chunmei Zhang, Dandan Wen, Fuyong Yue, Yuttana Intaravanne, Wei Wang, and Xianzhong Chen. Optical Metasurface Generated Vector Beam for Anticounterfeiting. Physical Review Applied, 10(3):034028, September 2018.

[18] Bicher Haj Ibrahim, Sami Ben Hatit, and Antonello De Martino. Angle resolved mueller polarimetry with a high numerical aperture and characterization of transparent biaxial samples. Appl. Opt., 48(27):5025-5034, Sep 2009.

[19] Angelo Pierangelo, Abdelali Benali, Maria-Rosaria Antonelli, Tatiana Novikova, Pierre Validire, Brice Gayet, and Antonello De Martino. Ex-vivo characterization of human colon cancer by mueller polarimetric imaging. Opt. Express, 19(2):1582-1593, Jan 2011.

[20] Radoslaw Kolkowski, Lucia Petti, Massimo Rippa, Clement Lafargue, and Joseph Zyss. Octupolar plasmonic meta-molecules for nonlinear chiral watermarking at subwavelength scale. ACS Photonics, 2(7):899-906, 2015 .

[21] E. Bernabeu and J.-J. Gil. An experimental device for the dynamic determination of mueller matrices. Journal of Optics, 16(3):139-141, 1985.

[22] R. M. A. Azzam. Photopolarimetric measurement of the mueller matrix by fourier analysis of a single detected signal. Opt. Lett., 2(6):148-150, Jun 1978.

[23] R. W. Collins and Joohyun Koh. Dual rotatingcompensator multichannel ellipsometer:̈̈Éinstrument design for real-time mueller matrix spectroscopy of surfaces and films. J. Opt. Soc. Am. A, 16(8):1997-2006, Aug 1999.

[24] Oriol Arteaga, Marta Baldrís, Joan Antó, Adolf Canillas, Esther Pascual, and Enric Bertran. Mueller matrix microscope with a dual continuous rotating compensator setup and digital demodulation. Applied Optics, 53(10):2236, April 2014.

[25] K. Ichimoto, Kazuya Shinoda, T. Yamamoto, and J. Kiyohara. Photopolarimetric measurement system of mueller matrix with dual rotating waveplates. Publ. Natl. Astron. Obs. Jap., 9:11, 2006. 
[26] P. S. Hauge. Mueller matrix ellipsometry with imperfect compensators. J. Opt. Soc. Am., 68(11):1519-1528, Nov 1978.

[27] Emanuel Chironi and Claudio Iemmi. Error analysis and compensation for a discrete dual rotating retarder mueller matrix polarimeter. Appl. Opt., 59(21):63686378, Jul 2020.

[28] G. Piller, L. Broch, and L. Johann. Experimental study of the systematic errors for a mueller matrix double rotating compensator ellipsometer. Physica status solidi $C$, 5(5):1027-1030, 2008.

[29] Razvigor Ossikovski. Differential matrix formalism for depolarizing anisotropic media. Opt. Lett., 36(12):23302332, Jun 2011.

[30] Razvigor Ossikovski and Antonello De Martino. Differential mueller matrix of a depolarizing homogeneous medium and its relation to the mueller matrix logarithm. J. Opt. Soc. Am. A, 32(2):343-348, Feb 2015.

[31] Peter Wilson. Chapter 7 - high speed video application. In Peter Wilson, editor, Design Recipes for FPGAs (Second Edition), pages 67-77. Newnes, Oxford, second edition edition, 2016.

[32] Ivana Sersic, Christelle Tuambilangana, Tobias Kampfrath, and A. Femius Koenderink. Magnetoelectric point scattering theory for metamaterial scatterers. Phys. Rev. B, 83:245102, Jun 2011.

[33] Ivana Sersic, Martin Frimmer, Ewold Verhagen, and A. Femius Koenderink. Electric and magnetic dipole coupling in near-infrared split-ring metamaterial arrays. Phys. Rev. Lett., 103:213902, Nov 2009.

[34] Julien Proust, Nicolas Bonod, Johan Grand, and Bruno Gallas. Optical Monitoring of the Magnetoelectric Coupling in Individual Plasmonic Scatterers. ACS Photonics, 3(9):1581-1588, September 2016.

[35] Weiqi Li, Chuanwei Zhang, Hao Jiang, Xiuguo Chen, and Shiyuan Liu. Depolarization artifacts in dual rotatingcompensator mueller matrix ellipsometry. Journal of $\mathrm{Op}$ tics, 18(5):055701, mar 2016.

[36] José Jorge Gil and Eusebio Bernabeu. Depolarization and polarization indices of an optical system. Optica Acta: International Journal of Optics, 33(2):185-189, 1986.

[37] Noah A. Rubin, Gabriele D'Aversa, Paul Chevalier, Zhujun Shi, Wei Ting Chen, and Federico Capasso. Matrix Fourier optics enables a compact full-Stokes polarization camera. Science, 365(6448):eaax1839, July 2019. 